\title{
Valence Electron States of Carbon Materials studied by TEM-SXES
}

\author{
M. Terauchi ${ }^{1}$
}

${ }^{1}$ IMRAM, Tohoku University, 2-1-1 Katahira, Aoba-ku, Sendai 980-8577, Japan.

$\mathrm{X}$-ray emission spectroscopy is widely used as a practical tool for compositional analysis and/or elemental mapping analysis in electron microscopes. The characteristic X-rays originate form electronic transitions from valence bands (VB, bonding electron states) to inner-shell electron levels inform us energy states of bonding electrons. Those X-ray energies are ranged in ultrasoft or soft X-ray region form about 0.01 to a few $\mathrm{keV}$. Thus, soft-X-ray emission spectroscopy (SXES) combined with electron microscopy should be the best way for evaluating bonding states of identified specimen areas of newly developed functional materials. For that purpose, we have introduced compact SXES instruments, which are composed of varied line spacing gratings combined with area detectors, to transmission electron microscopes (TEM) [1,2], electron-probe micro-analyzer [3] and scanning electron microscope [4].

Applications of TEM-SXES instrument to carbon and boron materials have revealed characteristic energy distribution of bonding electrons [5]. Figure 1 shows carbon K-emission (VB $\rightarrow \mathrm{K}$-shell) spectra of zeolite-Y template carbon (ZTC) [6]. Spectra of graphite and momomer- $\mathrm{C}_{60}$ (Mono.- $\mathrm{C}_{60}$ ) are also shown for comparison. As ZTC has a huge surface area of $4000 \mathrm{~m}^{2} / \mathrm{g}$, it is expected to be applied to fuel cell and electrode of rechargeable batteries. Electron diffraction pattern of this material shows broad circularly distributed intensities similar to that of amorphous carbon. However, C K-emission spectrum shows apparent structures. Those structure positions similar to those of momomer- $\mathrm{C}_{60}$ than those of graphite. This result indicates that ZTC is basically composed of curved grapheme, $\mathrm{sp}^{2}$, network with a similar curvature with that of a $\mathrm{C}_{60}$ cluster. This result is consistent with theoretical simulation. Additional structures at the top end and on the lower energy side tale of VB as indicated by arrows are similar characters with those of Diamond (not shown here). Thus, this indicates a presence of a certain amount of $\mathrm{sp}^{3}$ component in carbon network of ZTC examined.

As graphite has anisotropic covalent bonding nature with $\sigma\left(\mathrm{sp}^{2}\right)$ and $\pi\left(\mathrm{p}_{\mathrm{z}}\right)$ bondings, C K-emission of graphite should show anisotropic distribution. Furthermore, those emitted X-ray photons should be polarized. For examining the anisotropic emission, SXES measurements with changing a crystal orientation is useful, which is easily conducted by using a TEM based SXES instrument. Figure 2(a) shows C K-emission spectra of single-crystalline graphite obtained at different crystal settings. The right-hand-side end of the intensity distribution corresponds to the top of VB. Emission intensity I(9) at a take-off angle $\theta$ is written as $I(\theta)=I_{\pi} \cos ^{2} \theta+I_{\sigma}\left(1+\sin ^{2} \theta\right) / 2$, where $I_{\pi}$ and $I_{\sigma}$ are emission intensity distributions of $\pi$ - and $\sigma$-bonding states, respectively [7]. The take-off angle is an angle between an emission direction and (0001) plane of graphite. The intensity profiles of (1) and (2) are correspond to $\mathrm{I}=\mathrm{I}_{\pi}+0.5 \cdot \mathrm{I}_{\sigma}$ and $\mathrm{I}=0.18 \mathrm{I}_{\pi}:+0.91 \mathrm{I}_{\sigma}$, respectively. Intensity distributions of $\mathrm{I}_{\pi}$ and $\mathrm{I}_{\sigma}$ derived by using the equations are shown in the lower part of Fig. 2(b). It is clearly seen that I: distributes in the upper part and $I_{\sigma}$ distribute in the lower part of VB. The structures indicated by vertical lines of $\pi$-bonding states correspond to electronic states on Brillouin zone boundary at $\mathrm{P}$ and $\mathrm{Q}$, respectively. However, the effect of polarization of emitted X-ray photons is not included in this analysis, because reflectance for s-and p-polarizations is assumed to be the same. At the incidence angle of X-ray to the Au-surface, measured from the surface normal of the grating surface, was $86 \mathrm{deg}$. for this experiment. The values of reflectance for s- and p-polarizations are 0.88 and 0.70 , respectively [8]. Then, the modified equations 
for (1) and (2) in Fig. 2(a) are $0.88 \cdot I_{\pi}+0.35 \cdot I_{\sigma}$ and $0.13 \cdot I_{\pi}+0.73 \cdot I_{\sigma}$, respectively. Intensity distributions of $\mathrm{I}_{\pi}$ and $\mathrm{I}_{\sigma}$ derived by those corrected equations are shown in the upper part of Fig. 2(b). The peak intensity of $\sigma$-band compared to that of $\pi$-band is increased and becomes closer to theoretical result. This suggests that the polarization correction is presumably necessary for an accurate analysis of VB by using a grating spectrometer.

\section{References:}

[1] M Terauchi, H Yamamoto and M Tanaka, J. Electron Microscopy 50 (2001), 101.

[2] M Terauchi et al, Journal of Electron Microscopy 61 (2012), 1.

[3] H Takahashi et al, Microscopy and Microanalysis 19(Suppl.2) (2013), 1258.

[4] M Terauchi et al, Microscopy and Microanalysis, accepted.

[5] M Terauchi in "Transmission Electron Microscopy Characterization of Nanomaterials", ed. CSSR Kumar, (Springer-Verlag, Berlin Heidelberg) 284.

[6] K Nueangnoraj et al., CARBON 62 (2013), 455.

[7] E Tegeler et al, Phys. Stat. Sol. 84 (1977), 561.

[8] Hunter WR (2000) in "Vacuum Ultraviolet Spectroscopy", eds. Samson JA, and Ederer DL (Academic Press, San Diego) 234.

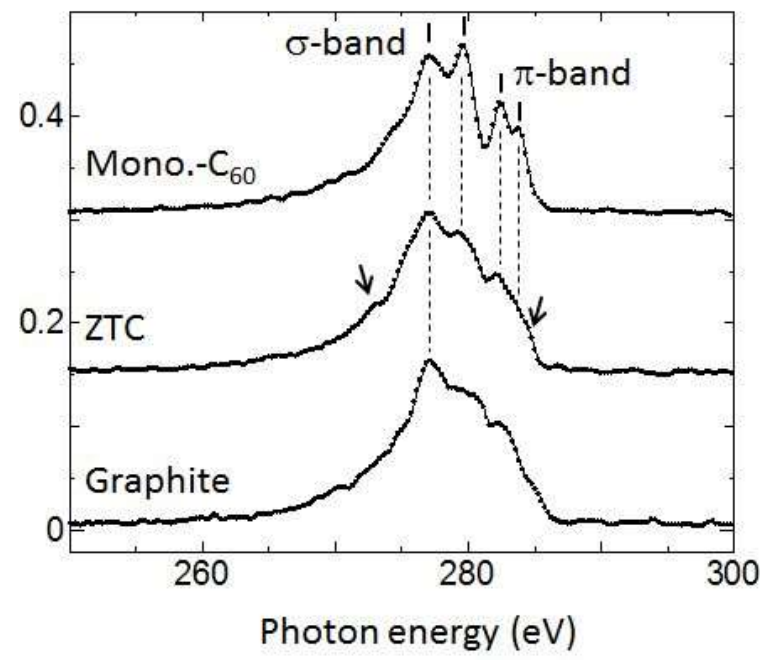

Figure 1. (a) C K-emission spectra of zeolite-Y template carbon (ZTC), monomer- $\mathrm{C}_{60}$ and graphite. ZTC shows a similar structure with those of Mono- $\mathrm{C}_{60}$ than those of graphite.
Figure 2. (a) C K-emission spectra of single-crystalline graphite obtained at different crystal settings. (b) Numerically derived $\pi$-bonding and $\sigma$-bonding state distributions from the two spectra in (a) without (lower) and with (upper) polarization correction.
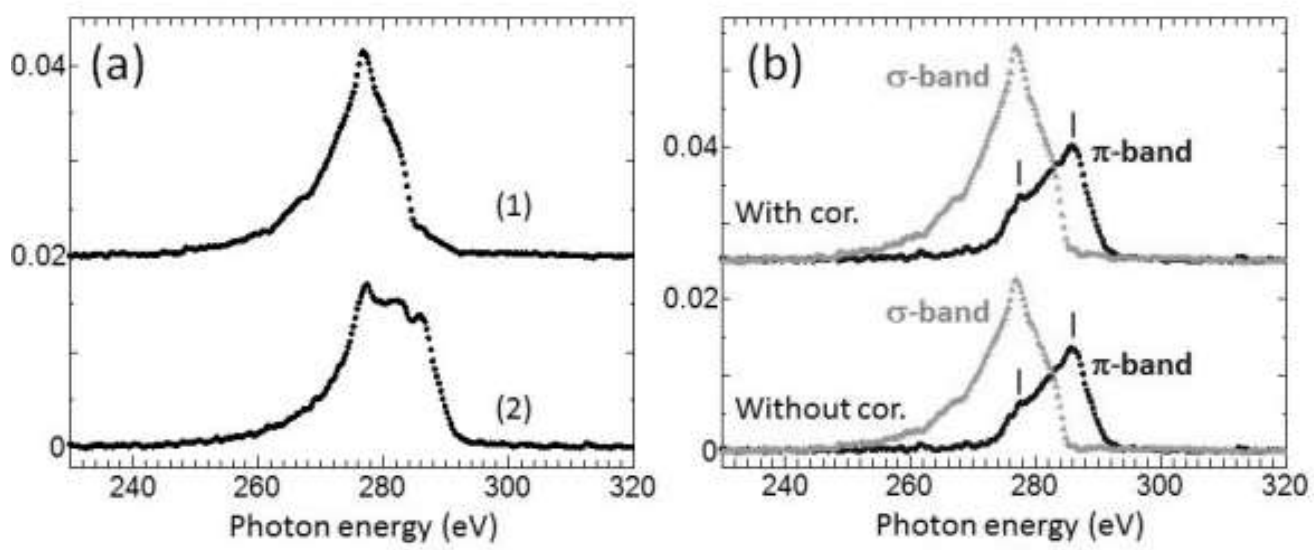